\title{
Inter-Fraction and Intra-Fraction Variation in the Absorbed-Dose Delivery during Interstitial High Dose Rate Brachytherapy-A Study Using MicroMOSFET In-Vivo Dosimeter
}

\author{
Seenisamy Ramapandian'1, Vivekanandan Nagarajan², Ashutosh Mukherji', \\ Parthasarathy Vedasoundaram ${ }^{1}$, K. S. Reddy ${ }^{3}$, Vivekanandam Singhavajala ${ }^{1}$, \\ Vijayaprabhu Neelakandan 1
}

\author{
${ }^{1}$ Department of Radiotherapy, RCC, JIPMER, Pondicherry, India \\ ${ }^{2}$ Department of Medical Physics, Cancer Institute, Chennai, India \\ ${ }^{3}$ Department of Oncology, MGMC \& RI, Puducherry, India \\ Email:nellaiseeni@gmail.com
}

How to cite this paper: Ramapandian, S., Nagarajan, V., Mukherji, A., Vedasoundaram, P., Reddy, K.S., Singhavajala, V. and Neelakandan, V. (2017) Inter-Fraction and Intra-Fraction Variation in the Absorbed-Dose Delivery during Interstitial High Dose Rate Brachytherapy-A Study Using MicroMOSFET In-Vivo Dosimeter. International Journal of Medical Physics, Clinical Engineering and Radiation Oncology, 6, 445-456. https://doi.org/10.4236/ijmpcero.2017.64040

Received: October 10, 2017

Accepted: November 26, 2017

Published: November 29, 2017

Copyright $\odot 2017$ by authors and Scientific Research Publishing Inc. This work is licensed under the Creative Commons Attribution International License (CC BY 4.0).

http://creativecommons.org/licenses/by/4.0/ (c) (i) Open Access

\begin{abstract}
Background: The delivered dose has to be checked and verified with planned dose since precise and accurate dose delivery is essential in Brachytherapy. Sources of uncertainty during Brachytherapy are intra-fraction, inter-fraction and inter-application variations. In-vivo dosimetry is the direct method to monitor the radiation dose delivered to a patient during radiotherapy. In this study, assessment of the inter-fraction and intra-fraction variations in the interstitial Brachytherapy was done with microMOSFET. Aim: To analyze the inter-fraction variations in dose delivery during interstitial HDR Brachytherapy and to compare the measured point dose with the TPS-calculated point dose, intra-fraction variation, using the microMOSFET in-vivo dosimeter. Materials and Methods: From May 2014 to February 2016, 22 patients with Head and Neck cancers and 8 patients with Soft-Tissue Sarcomas (STS) were selected for this study. All these patients underwent CT imaging more than 24 hours after the application. Brachyvision 3DTPS and GammaMed Plus iX HDR unit were used for treatments. MicroMOSFET in-vivo dosimeter after calibration was used for the measurements of dose inside the treated volume. Intra \& Inter-fraction variations were analyzed and reported. Results: The SD of inter-fraction variation among 22 Head \& Neck patients ranges from $2.14 \%$ to $14.26 \%$. Minimum \& maximum dose variation with first fraction dose of patients ranged from $-22.33 \%$ to $+26.71 \%$ and the mean doses were $-6.42 \%$ to $+19.76 \%$. Differences of TPS dose and microMOSFET measured first fraction dose, intra-fraction variation, ranged from $-12.36 \%$ to $+5.05 \%$. The $\mathrm{SD}$ of inter-fraction variation
\end{abstract}


for 8 STS patients was from $2.81 \%$ to $14.43 \%$. Minimum and maximum doses vary from $-38.72 \%$ to $+25.74 \%$ and mean dose varies from $-21.5 \%$ to $+12.53 \%$. Differences of point doses of TPS and measured, intra-fraction variation, were from $-5.86 \%$ to $4.88 \%$. Conclusions: MicroMOSFET has the potential to minimize the gross errors during multi-fractionated Interstitial Brachytherapy. Edema, applicator displacements and placement of microMOSFET are the main influencing factors for inter-fraction uncertainty in dose delivery. Re-planning with re-simulated images should be considered whenever the microMOSFET readings vary more than $\pm 10 \%$ of the planned dose inside the CTV measured in two successive fractions.

\section{Keywords}

MicroMOSFET, Interstitial Brachytherapy, MicroMOSFET in Brachytherapy, In-Vivo Dosimetry in Brachytherapy, Dose Verification in Brachytherapy

\section{Introduction}

The advantage of Brachytherapy is the higher degree of dose localization in the target volume with better dose sparing of normal tissue [1]. Interstitial Brachytherapy, an invasive form of Brachytherapy, offers a flexible way of customized treatment for the patient, and is usually chosen as a radical treatment for the early-stage Head \& Neck cancers, Breast Cancers and for Soft Tissue Sarcoma (STS) [2]. Flexible tubes are commonly used as source guides during treatments of these sites.

The quality control in Brachytherapy involves determination of source strength, positional accuracy and the TPS calculation accuracy [3]. As per the ICRU 89, the sources of uncertainty, in general, include intra-fraction, inter-fraction and inter-application variations [4]. It defines the intra-fraction variations as those occurring between imaging/dose planning and absorbed dose delivery, the inter-fraction as those occurring between two fractions based on the same imaging and dose planning, and also when several fractions are delivered with same applicator insertion.

The AAPM Task Group report No. 62 defines the in-vivo dosimetry as the direct method "to monitor the radiation dose delivered to a patient during radiotherapy" [5]. Thus, in vivo dosimeter has the potential to verify that the actual dose is delivered during Brachytherapy and all the errors, including inter-fraction, intra-fraction and inter-application variations, could be determined.

In Brachytherapy, the dosimeter needs to be placed near or inside the treated volume as the dose gradient is larger and dose decreases rapidly with distance [6]. Thus, the Brachytherapy in-vivo dosimeters need to be small in their size in order to fit inside the applicators and to be placed directly inside the body cavities. Some of the in-vivo dosimeters like TLD, MOSFET, diodes, diamond detector and scintillation detector were compared and studied for their effectiveness by 
Lambert et al. [7] for Brachytherapy. The intra-fraction and inter-fraction dose delivery uncertainties during Interstitial Brachytherapy using flexible catheters are measured and analyzed in this study.

Metal Oxide Semiconductor Field Effect Transistor (MOSFET) used in this study is an insulated gate field effect transistor with a floating gate applicable in radiation dosimetry [8]. MOSFETs are used as an in-vivo dosimeter in Teletherapy due to their compact size, wireless design, dose rate independence, energy independence and beam geometry independence characters [9]. MOSFET dosimeters do not require post-exposure processing and dose measurements are done immediately after irradiation. Specially-designed microMOSFETs using Thomson-Neilson probes (M/s Best Medicals Canada) are available for Brachytherapy [10]. Change in threshold voltage of control gate bias of the microMOSFET to achieve the drain current is directly related to the amount of radiation exposure given. MOSFETs need to be calibrated using the relevant radiation before clinical use. The ideal distance for consistent reading of MOSFET is $2 \mathrm{~cm}$ to $5 \mathrm{~cm}$ from the Ir-192 radioisotope in water [11], where the accuracy of the measurements was within $5 \%$.

The delivered dose has to be checked and verified with planned dose since precise and accurate dose delivery is essential in Brachytherapy. In this study, assessment of the inter-fraction and intra-fraction variations in the interstitial Brachytherapy was done with microMOSFET.

\section{Materials and Methods}

From May 2014 to February 2016, twenty two patients with Head and Neck cancers \& eight patients with Soft-Tissue Sarcomas (STS) and who were planned to be treated with radical intent that include HDR Brachytherapy using flexible tube interstitial implants were selected for this study (Table 1). All these patients underwent CT imaging for 3D Brachytherapy planning, more than 24 hours after the application to allow the healing of edema in the implanted volume. The prescription dose to the Clinical Target Volume (CTV) of the Head and Neck Cancers was $21 \mathrm{~Gy}$ delivered in seven fractions. The prescription dose in the patients with STS was decided on case to case basis. For 3D planning BrachyVision 3D TPS version 10.0.0 (M/s Varian Medical Systems, UK) was used and treatments were executed for all these patients with 24-channel GammaMed Plus iX HDR treatment unit (M/s Varian Medical Systems, UK). The dose calculations were done as per the AAPM TG 43 protocol.

Commercially-available microMOSFET in-vivo dosimeter (Standard) Model TN-502RDM (M/s Best ${ }^{\circledast}$ Medical Canada) was used in this study. The microMOSFET is useful in Brachytherapy as it fits down in a 6-French catheter, which is commonly used in interstitial Brachytherapy. All dose measurements were done inside the treated volume, by inserting the flex in the last catheter. Last catheter was housing the microMOSFET during measurement after that used for completing the remaining treatment portion. The microMOSFET was operated 
Table 1. Patient characteristics.

\begin{tabular}{lccc}
\hline & Factors & Numbers & $\%$ \\
\hline \multirow{2}{*}{ Age } & Mean & $53(30-84)$ & \\
& Median & $50(30-84)$ & $53 \%$ \\
Sex & Male & 16 & $47 \%$ \\
& Female & 25 & $36 \%$ \\
Ca Tongue & Ca Lip & 15 & $7 \%$ \\
Tumor site & Ca Floor of Mouth & 3 & $5 \%$ \\
& Ca Buccal Mucosa & 2 & $5 \%$ \\
& Soft Tissue Sarcoma & 8 & $27 \%$ \\
Catheters & Ca Cervix & 11 & \\
& Mean & $11(5-15)$ & \\
Interstitial Applications & $12(5-15)$ & \\
\hline
\end{tabular}

in the standard setting with standard bias in this study, which has a nominal sensitivity of $1 \mathrm{mV} / \mathrm{cGy}$. This study is a cross sectional dosimetric one and the outcome variable is reported as $\mathrm{SD}$.

\subsection{Interstitial Brachytherapy with Flexible Tube Catheters}

Head \& Neck cancers and Soft Tissue Sarcoma patients were selected as per our institute protocol for the multi-catheter interstitial Brachytherapy procedure. The procedure was performed under general or spinal anesthesia, and implants were done with Varian flexible tube catheters by the treating physician. Number of catheters and planes depended on the size, shape and location of the tumor (Figure 1). Patients were imaged using CT, 24 hours after the insertion procedure. CT Slices of $1-3 \mathrm{~mm}$ cuts were preferred for accurate reconstruction of the catheters. CT images were transferred to the Varian Brachyvision 3D treatment planning system through DICOM for 3D planning. Planning was done as per CTV delineated by the radiation oncologist. Dwell positions were decided based on the CTV. Dose prescription was $21 \mathrm{~Gy}$ in 7 fractions for head \& neck cases; while in sarcoma cases, total dose varied according to intent (post-op/recurrent/radical) and in this study only the first seven fractions for these cases have been assessed whenever the regime exceeded seven fractions. Treatment plan was evaluated using the coverage of CTV with D90 and D2cc to the Organs at Risk (OAR). After the approval, plans were exported to the HDR unit through DICOM. All the treatments were executed in GammaMed Plus iX HDR unit.

\subsection{Calibration of MicroMOSFET with Flexible Tube Catheters}

Five microMOSFET probes were used in this study. All microMOSFETs were calibrated in Ir-192 source with known calculated dose before their clinical use 


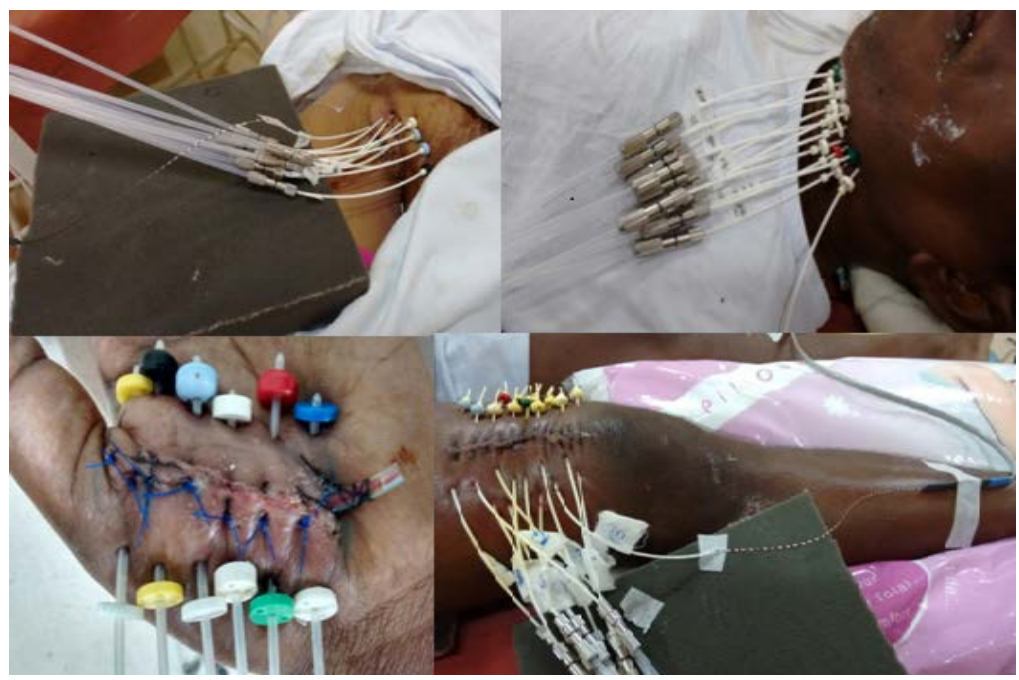

Figure 1. Flexible tube interstitial brachytherapy implant.

in dose measurements. As the physical characteristic of microMOSFET with Ir-192 is sensitive to the distance, calibrations need special attention [9]. Zilio et al. [12] measured the absolute depth dose rates of Ir-192 with microMOSFET in water phantom. Though the study of microMOSFET with Ir-192 in water phantom is proven one, we preferred to do the calibration in modeling wax phantom with multiple flexible tube catheters. Instead of calibrating microMOSFET at each different distance, we tried to mimic the clinical setup to get the collective readings of the multiple catheters at different distance and planes. Wax mould with equidistant of $1 \mathrm{~cm}$ placed flexible catheters in two planes was used for calibration of flexible tubes.

Six flexible catheters were fixed in each plane with a distance of $1 \mathrm{~cm}$ between each plane (Figure 2). The microMOSFET flex was fixed inside the last catheter at a distance of $4.5 \mathrm{~cm}$ from its tip, taking care that the measurement point still lies inside the CTV. The entire setup then was imaged by a CT scan and 3D planning was done for $100 \mathrm{cGy}$ at microMOSFET position, with equal dwell time. Plan was executed in the HDR unit. Calibrations were repeated three times for each microMOSFET flex for all five microMOSFETs and the average was taken as a calibration factor for flexible tubes. Linearity of the microMOSFETs was also checked with doses varying from $20 \mathrm{cGy}, 50 \mathrm{cGy}, 100 \mathrm{cGy}, 200 \mathrm{cGy}, 300 \mathrm{cGy}$ and 500 cGy. Dose-rate dependency was checked with different activities of the Ir-192 sources at various points of time. The angular dependency was taken care by keeping the probes face the upper plane during the measurements.

\subsection{Measurements of Inter-Fraction Variation Using MicroMOSFET}

The last catheter of each plan was used for the placement of microMOSFET. The position of the microMOSFET was in the centre of the active length of the last catheter, which varied from patient to patient. Distance from the tip of the applicator was $1-5 \mathrm{~cm}$ for microMOSFET placement for different patients. The 


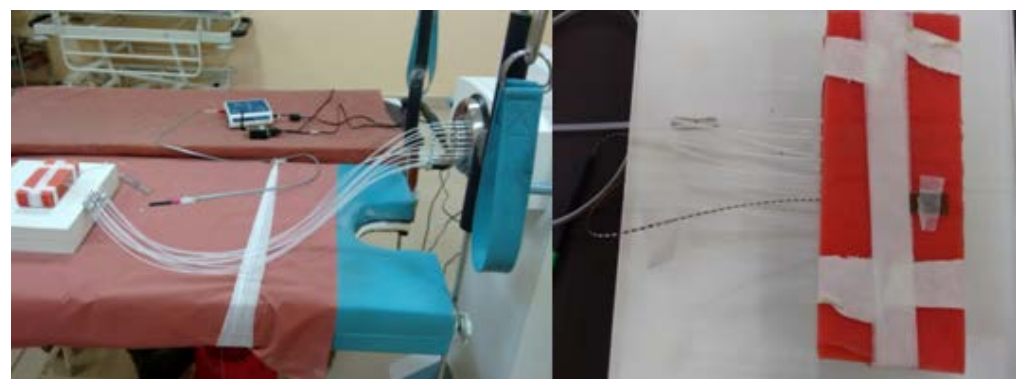

Figure 2. Calibration of microMOSFET with flexible tubes in Ir-192.

first fraction position, where the microMOSFET was exiting from the catheter proximal position, was marked and same probe was reproduced for the remaining fractions of that particular plan. microMOSFET probe is pasted with the catheter to fix the position during the measurement. Before starting the exposure, microMOSFET threshold values were noted. Change in the pre and post treatment threshold voltage values is thought to be directly proportional to the exposure received by the microMOSFET. First fraction reading was taken as a baseline value, $100 \%$. Remaining measurements for the particular plan were compared with the baseline value. Variations in the inter-fraction microMOSFET values were analyzed in this study.

\subsection{Intra-Fraction Variation Measurements Using MicroMOSFET}

In addition to inter fraction variation, we have analyzed the intra-fraction variations by comparing the microMOSFET measured point dose during the first fractionation session with that of the TPS calculated point dose during planning. Advancements in Teletherapy have lot of options to verify the plan before as well as during the delivery. However, Brachytherapy has limited options. Comparison of point doses with microMOSFET can be an instantaneous, simple and accurate solution for independent verification of the Brachytherapy plan. For this study we have made additional plan to mimic the delivered plan, but without the last catheter. Last catheter was removed in all plans, that used to be the placement of microMOSFET probe and calculations were done keeping the remaining dwell positions and their dwell times same. New reference point is created for each plan which exactly matching with the microMOSFET position in the delivered plan. These TPS calculated point doses were noted and compared with microMOSFET measured point doses for each patient.

\section{Results}

Twenty two flexible catheter interstitial implanted patients' of Head \& Neck cancers and eight STS patient's data have been taken for analysis. Only the first seven fractions were considered for analysis, though some of STS patients received more than seven fractions. All patients successfully completed radiation treatment.

The calibration factor of microMOSFET with flexible tube in Ir-192 was found 
to be $0.8547 \mathrm{cGy} / \mathrm{mV}$. Figure 3 shows the SD of inter-fraction ranges from 2.14 to 14.26 for all 22 Head \& Neck cancer patients.

Variations of the microMOSFET measurement ranged from $-22.3 \%$ to $+26.71 \%$ with first fraction value for all patients. In minimum side, 4/22 patients had more than $-10 \%$ variation within the treatment regime rest of them, $18 / 22$, had less than $-10 \%$. But the maximum variation happened in $8 / 22$ incidences above $+10 \%$ and the other $14 / 22$ were below $+10 \%$. However, the mean variation ranges from $-6.42 \%$ to $+19.76 \%$ in comparison with first fraction dose. $3 / 22$ patient's mean dose were beyond $10 \%$ variation. Figure 4 compares the difference of the minimum, maximum and mean microMOSFET values with first fraction value in percentage of each patient.

First fraction values were considered as a base line value for point dose and compared with the TPS calculated point dose on the same microMOSFET position for intra-fraction variations. Figure 5 shows the intra-fraction variations, which vary from $-12.36 \%$ to $+5.05 \%$. $5 / 22$ patients had the intra-fraction variations more than $\pm 5 \%$.

SD of inter-fraction variation ranges from 2.81 to 14.43 for 8 STS patients (Figure 6).

Figure 7 compares the minimum and maximum doses vary from $-38.72 \%$ to $25.74 \%$ for all the 8 patients. And the mean doses were $-21.5 \%$ to $+12.53 \% .3 / 8$ patients had more than $\pm 20 \%$ variation and for the rest $5 / 8$ patients the variation was less than that, $\pm 15 \%$.

Differences of point doses for all 8 patients were from $-5.86 \%$ to $4.88 \%$. Except one patient, the intra-fraction variations were less than 5\% (Figure 8).

\section{Discussion}

From our study we found that the inter-fraction variations measured with microMOSFET in the interstitial implants with flexible catheter is high. The measured dose values fluctuated during successive fractions when compared with the

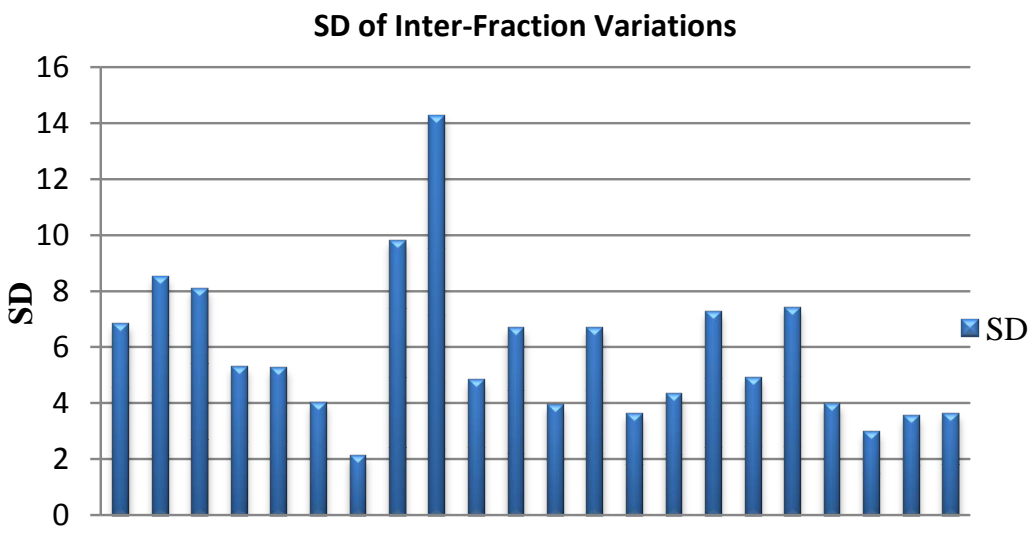

Pt Pt Pt Pt Pt Pt Pt Pt Pt Pt Pt Pt Pt Pt Pt Pt Pt Pt Pt Pt Pt Pt

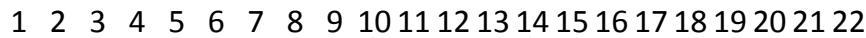
Head \& Neck Patients

Figure 3. SD of inter-fraction variations for head \& neck patients. 
Fraction1 Vs Min, Max \& Mean Delivered Doses in \%

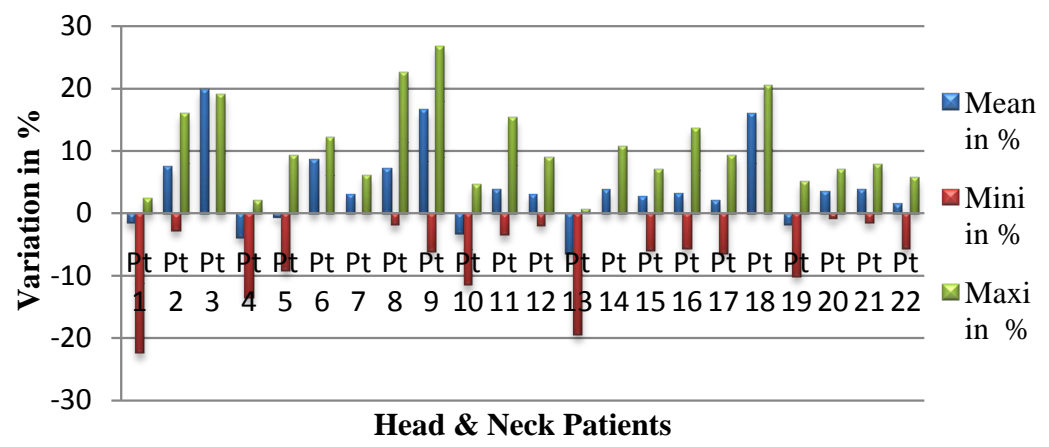

Figure 4. Minimum, maximum and mean doses with first fraction doses for head \& neck patients in percentage.

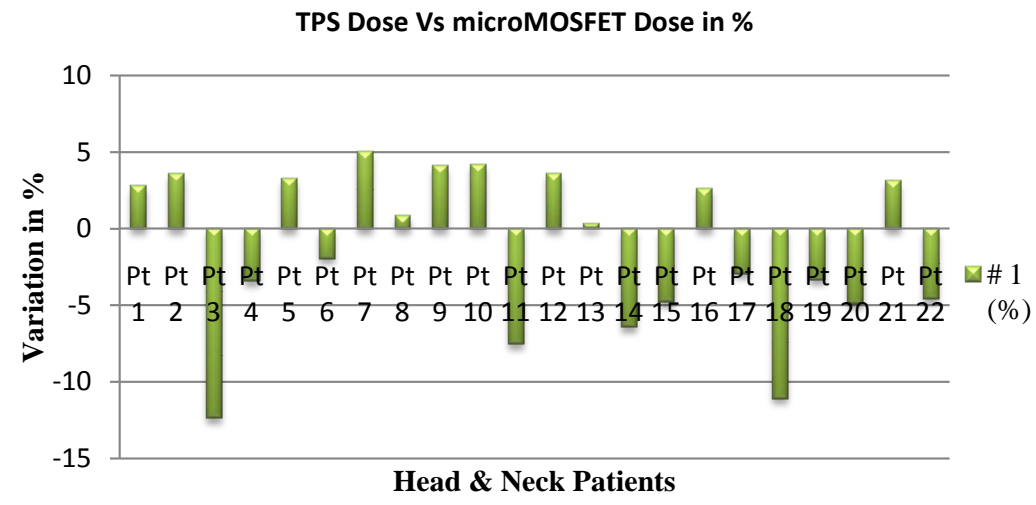

Figure 5. Intra-fraction variations of head \& neck patients in percentage.

\section{SD of Inter-fraction variations}

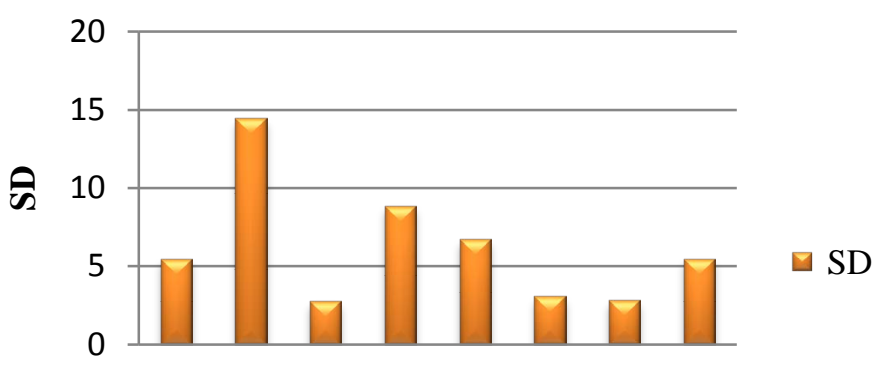

Pt 1 Pt 2 Pt 3 Pt 4 Pt 5 Pt 6 Pt 7 Pt 8

STS patients

Figure 6. SD of inter fraction variations for STS implant.

first fraction value. For some cases minor displacement of the catheters could have changed the peripheral doses but not the central part of the CTV. Moreover the movement of the catheter can happen in any direction i.e., along cranio-caudal, dorso-ventral and/or lateral. And this cumulative effect may nullify individual variations to some extent.

For Ca. Tongue patients, variations happened due to edema as well as the displacement of the applicator. Being a moving organ, reproducibility of the catheter 
Fraction1 Vs Min, Max \& Mean Delivered Doses in \%

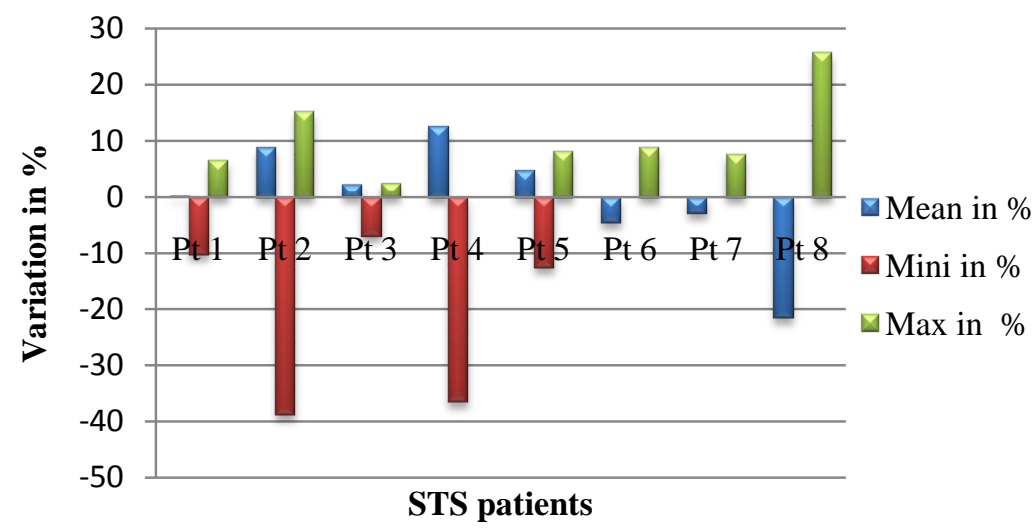

Figure 7. Minimum, maximum and mean values with first fraction values for STS patients in percentage.

TPS dose vs microMOSFET dose in \%

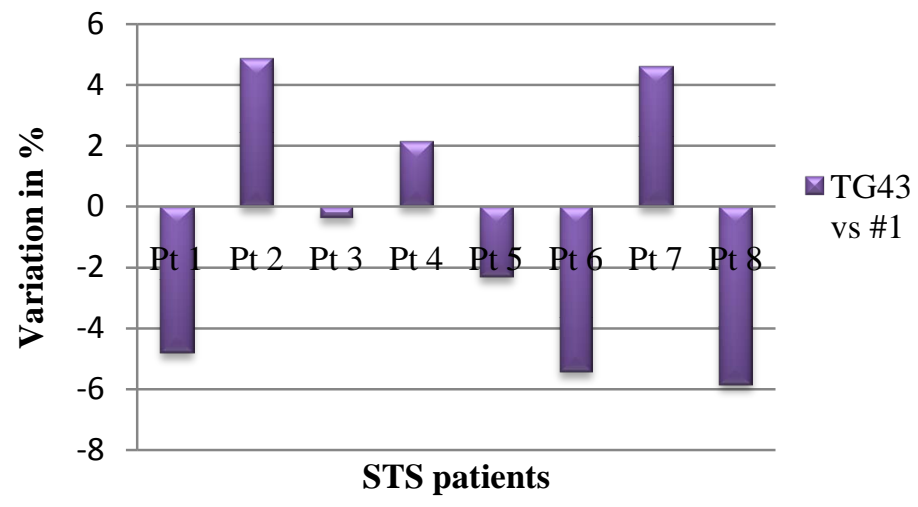

Figure 8. Intra-fraction variations of STS in percentage.

position and microMOSFET placement could be a tough task. Since lip also a moving organ, applicator movement likely happened. Moreover CTV volume also quite small, minor change in the applicator position leads to significant variation. For Ca Buccal Mucosa, applicator displacement may be comparatively less. But edema and changes in the tissue shrinkage may cause the variation. Applicator position, edema and tissue shrinkage may affect the delivered dose in Ca Floor of Mouth patients.

In STS cases variation ranged from low to high depending on the size and location of the tumor. Being superficial tumor, small displacement of applicator resulted in significant differences.

Rey et al. [13] in their study concluded that there was a decrease in the dose delivered during the length of the treatment in gynecological interstitial applications and suggested re-planning based on repeat CT scans before each or alternative fractions. In our study for flexible catheter based interstitial Brachytherapy, the planning CT scan was done 1 or 2 days after the implant. This had the beneficial effect of reduced tissue edema and hence a reduction in the effect of 
tissue volume difference due to edema between planning and delivery of treatment. However there was a variation in the delivered dose which could be because of reduced edema. Edema, applicator movement and placement of microMOSFET may have played a major role in affecting the delivered dose over the entire fractions significantly. Edema shrinkage causes the flexible catheters to come close to each other and would proportionally increase the dose to the measuring position.

Kandasamy et al. [14] observed inter-fraction errors frequently in the interstitial implants and recommended imaging before each fraction and re-planning based on that. In-vivo dosimetry with microMOSFET can be alternative for pre-treatment CT method with the added advantage of instant verification, convenient to patient, save the quality time of man power and no additional exposure unlike the multiple CT.

Takenaka et al. [15] investigated needle applicator movements in prostate cancers with daily CT and concluded that the displacement of the needles gradually increased with time. They suggested additional margin in the cranial direction instead of changing dwell positions. We also found that there is a variation in the delivered dose for flexible tube interstitial implant in Head \& Neck cancer patients which could be because of applicator displacement due to tissue shrinkage during the treatment process itself.

Onoe et al. [16] found the solution to overcome the cranio-caudal displacement of needles with the help of fully stretched elastic tape. They pasted the tape with the template assembly on the patient. This method drastically cut down the D90 of CTV within $\pm 5 \%$ in contrast with their earlier study of $35 \%$ to $40 \%$. Kandasamy et al. [14] suggested piece of micropore paper pasted on each applicator can minimize the displacement significantly. We also used the same method to arrest the applicator movement at certain extent.

The complex head \& neck anatomy frequently request the flexible implanted catheters in an irregular shape, so the microMOSFET position can be maintained but the direction facing the radiation was a challenging one. Khinikar et al. [11] found the angular dependence is $\pm 6 \%$ and angular effect is predominant in complex geometry like Head \& Neck than the relatively simple one like gynecological application.

Qi et al. [17] studied the sensitivity of microMOSFET with distance from the Ir-192. Their result shows that the variation is $7 \%$ for distance of $1-5 \mathrm{~cm}$. If the volume of flexible catheter implant is large then distance may be a contributing factor for dose variation.

Velmurugan et al. [18] suggested that the variation is significant and CT imaging for each fraction should be done to minimize the error in gynecological patients. CT/MRI imaging before each fraction can be an effective way to find the tumor shrinkage, edema and applicator displacement.

Instead of analyzing each fraction variation, each catheter variation for all fractions can find out the erratic applicator and give more accurate solution. This study can be continued in that direction. The microMOSFET measured doses may 
be compared with Model-Based Dose-Calculation Algorithm (MBDCA) doses for entire study. Differences between the microMOSFET dose and TPS with MBDCA doses may narrow down further.

\section{Conclusions}

There is no specific pattern in the microMOSFET measurements for flexible catheter interstitial Brachytherapy at various fractions. MicroMOSFET will be an effective dosimeter to monitor the inter-fraction and intra-fraction variation. With such monitoring, re-planning for every Brachytherapy session can be avoided and thus it can minimize the radiation exposure due to repeated pre-treatment CT and save quality time for repeat CT and re-planning. Edema, applicator displacement and placement of microMOSFET are the main influencing factors for the inter-fraction variations. Whenever the microMOSFET values deviate more than $\pm 10 \%$ during the successive two fractions, re-simulation and re-planning may be considered. Instead of analyzing each fraction variation, each catheter variation for all fractions can give more accurate solution to find out the displacement of catheter. This study can be continued in that direction.

For intra-fraction variations, comparison of TPS calculated point doses with microMOSFET measured point dose was done. Delivered dose, as measured with microMOSFET, is known immediately and due to its simple usage, microMOSFETs is found to be useful in-vivo dosimeter in a busy clinic as well as an independent verification of the Brachytherapy plan. microMOSFET can be used as a quality control tool to minimize the gross errors in the delivered dose during interstitial Brachytherapy.

\section{References}

[1] Nath, R., Rivard, M.J., De Werd, L.A., et al. (2016) Guidelines by the AAPM and GEC-ESTRO on the Use of Innovative Brachytherapy Devices and Applications: Report of Task Group 167. Medical Physics, 43, 3178-3205. https://doi.org/10.1118/1.4951734

[2] Takácsi-Nagy, Z., Martínez-Mongue, R., Mazeron, J.J., Anker, C.J. and Harrison, L.B. (2016) American Brachytherapy Society Task Group Report: Combined External Beam Irradiation and Interstitial Brachytherapy for Base of Tongue Tumors and Other Head and Neck Sites in the Era of New Technologies. Brachytherapy, 16, 44-58. https://doi.org/10.1016/j.brachy.2016.07.005

[3] Palmer, A., Bradley, D. and Nisbet, A. (2012) Physics-Aspects of Dose Accuracy in High Dose Rate (HDR) Brachytherapy: Source Dosimetry, Treatment Planning, Equipment Performance and In Vivo Verification Techniques. Journal of Contemporary Brachytherapy, 4, 81. https://doi.org/10.5114/jcb.2012.29364

[4] Potter, R., Kisistis, C. and Erickson, B. (2013) Prescribing, Recording and Reporting Brachytherapy for Cancer of the Cervix. JICRU Report, 13, 1-2.

[5] York, E. (2005) Diode In Vivo Dosimetry for Patients Receiving External Beam Radiation Therapy: Report of the American Association of Physicists in Medicine (AAPM) Task Group 62. Medical Physics Publishing, Madison.

[6] Tanderup, K., Beddar, S., Andersen, C.E., Kertzscher, G. and Cygler, J.E. (2013) In 
Vivo Dosimetry in Brachytherapy. Medical Physics, 40, Article Number: 070902. https://doi.org/10.1118/1.4810943

[7] Lambert, J., Nakano, T., Law, S., Elsey, J., McKenzie, D.R. and Suchowerska, N. (2007) In Vivo Dosimeters for HDR Brachytherapy: A Comparison of a Diamond Detector, MOSFET, TLD, and Scintillation Detector. Medical Physics, 34, 1759-1765. https://doi.org/10.1118/1.2727248

[8] Tarr, N.G., Mackay, G.F., Shortt, K. and Thomson, I. (1997) A Floating Gate MOSFET Dosimeter Requiring no External Bias Supply. Fourth European Conference on Radiation and Its Effects on Components and Systems, 1997, Cannes, 15-19 September 1997, 277-281.

[9] Gopiraj, A., Billimagga, R.S. and Ramasubramanian, V. (2008) Performance Characteristics and Commissioning of MOSFET as an In-Vivo Dosimeter for High Energy Photon External Beam Radiation Therapy. Reports of Practical Oncology and Radiotherapy, 13, 114-125. https://doi.org/10.1016/S1507-1367(10)60001-6

[10] Ramaseshan, R., Kohli, K.S., Zhang, T.J., et al. (2004) Performance Characteristics of a microMOSFET as an In Vivo Dosimeter in Radiation Therapy. Physics in Medicine and Biology, 49, 4031. https://doi.org/10.1088/0031-9155/49/17/014

[11] Kinhikar, R.A., Sharma, P.K., Tambe, C.M. and Deshpande, D.D. (2006) Dosimetric Evaluation of a New One Dose MOSFET for Ir-192 Energy. Physics in Medicine \& Biology, 51, 1261. https://doi.org/10.1088/0031-9155/51/5/015

[12] Zilio, V.O., Joneja, O.P., Popowski, Y., Rosenfeld, A. and Chawla, R. (2006) Absolute Depth-Dose-Rate Measurements for an Ir192 HDR Brachytherapy Source in Water using MOSFET Detectors. Medical Physics, 33, 1532-1539. https://doi.org/10.1118/1.2198168

[13] Rey, F., Chang, C., Mesina, C., Dixit, N., Teo, B.K. and Lin, L.L. (2013) Dosimetric Impact of Interfraction Catheter Movement and Organ Motion on MRI/CT Guided HDR Interstitial Brachytherapy for Gynecologic Cancer. Radiotherapy and Oncology, 107, 112-116. https://doi.org/10.1016/j.radonc.2012.12.013

[14] Kandasamy, S., Reddy, K.S., Nagarajan, V., Vedasoundaram, P. and Karunanidhi, G. (2013) Dosimetric Impact of Inter-Fraction Variation in Interstitial HDR Brachytherapy. International Journal of Medical Physics, Clinical Engineering and Radiation Oncology, 2, 111. https://doi.org/10.4236/ijmpcero.2013.24015

[15] Takenaka, T., Yoshida, K., Ueda, M., et al. (2012) Assessment of Daily Needle Applicator Displacement during High-Dose-Rate Interstitial Brachytherapy for Prostate Cancer using Daily CT Examinations. Journal of Radiation Research, 53, 469-474.

[16] Onoe, T., Nose, T., Yamashita, H., Yoshioka, M., Toshiyasu, T., Kozuka, T., Oguchi, M. and Nakagawa, K. (2013) High-Dose-Rate Interstitial Brachytherapy for Gynecologic Malignancies-Dosimetric Changes during Treatment Period. Journal of Radiation Research, 54, 663-670. https://doi.org/10.1093/jrr/rrs130

[17] Qi, Z.Y., Deng, X.W., Cao, X.P., Huang, S.M., Lerch, M. and Rosenfeld, A. (2012) A Real-Time in Vivo Dosimetric Verification Method for High-Dose Rate Intracavitary Brachytherapy of Nasopharyngeal Carcinoma. Medical Physics, 39, 6757-6763. https://doi.org/10.1118/1.4758067

[18] Velmurugan, T., Sukumar, P., Krishnappan, C. and Boopathy, R. (2010) Study of Dosimetric Variation Due to Interfraction Organ Movement in High Dose Rate Interstital (MUPIT) Brachytherapy for Gynecologic Malignancies. Polish Journal of Medical Physics and Engineering, 16, 85-95. 